\title{
Density dependent harvesting of a logistic population in a slowly varying environment T. Grozdanovski ${ }^{1} \quad$ J. J. Shepherd ${ }^{2} \quad$ A. Stacey ${ }^{3}$
}

(Received 8 December 2009; revised 22 February 2010)

\begin{abstract}
We apply a multiscale method to construct general analytic approximations for the solution of a harvested logistic system, where the system parameters vary slowly in time. Such approximations are a useful alternative to numerical solutions and are applicable to a range of parameter values. We consider two situations: subcritical harvesting, where the population survives; and supercritical harvesting, where it is driven to extinction. These approximations give excellent agreement with the numerical solutions of test cases.
\end{abstract}

\section{Contents}

1 Introduction

C33

2 The multiscale harvesting equation

C36

http://anziamj . austms.org.au/ojs/index.php/ANZIAMJ/article/view/2271 gives this article, (c) Austral. Mathematical Soc. 2010. Published February 26, 2010. ISSN 1446-8735. (Print two pages per sheet of paper.) 
3 Perturbation analysis

3.1 Subcritical harvesting . . . . . . . . . . . . C38

3.2 Supercritical harvesting . . . . . . . . . . . . . C40

4 Comparison with numerical solutions

C41

5 Discussion

$\mathrm{C} 43$

References

C46

\section{Introduction}

Consider a spatially homogenous single species population that, in isolation, changes according to a logistic law. When we harvest this at a rate proportional to the population, the rate of change in the population over time

$$
\frac{d P(T)}{d T}=R(T) P(T)\left(1-\frac{P(T)}{K(T)}\right)-E(T) P(T), \quad P(T=0)=P_{0} .
$$

Here $P(T)$ is the population (or population density) at times $T \geqslant 0, P_{0}$ is the initial population, while $R(T), K(T)$ and $E(T)$ are the time-varying growth rate, carrying capacity and harvesting effort respectively. All of these are positive quantities, and in the simplest case are constants $[8,2]$. The first term on the right hand side of (1) arises from logistic growth, while the second, proportional to $\mathrm{P}(\mathrm{T})$, arises from harvesting.

In the simple case where $R, K$ and $E$ are positive constants: if $E<R$, the population $\mathrm{P}(\mathrm{T})$ tends to $\mathrm{K}(\mathrm{R}-\mathrm{E}) / \mathrm{R}$, a positive value less than $\mathrm{K}$, as $T \rightarrow \infty$; whereas if $E>R$, the population tends to zero as $T \rightarrow \infty$, that is, the population is driven to extinction [8, Sec. 1.6]. Thus, $E=R$ is a critical value, and we term $\mathrm{E}<\mathrm{R}$ subcritical harvesting, while $\mathrm{E}>\mathrm{R}$ corresponds to supercritical harvesting. 
In the real world, $\mathrm{R}, \mathrm{K}$ and $\mathrm{E}$ vary, as in (1) and the question arises as to the effect of this variation on the evolving population. This matter has been examined in a number of publications, in various ways. Beddington and May [1] discuss the effects of random variation of the environment (via $R, K$ and E) with reference to the constant parameter case. No explicit solutions for varying parameters are given. Rosenblat [9] considers a periodically varying environment, and shows that this results in periodic 'equilibrium' states, with the location of the change from subcritical to supercritical harvesting fluctuating due to this variation. Legovic and Peric [7] use a form of the exact solution of (1) (see (6)) to obtain limiting forms of the population when $\mathrm{K}$ varies periodically, while $\mathrm{R}$ and $\mathrm{E}$ are kept constant. Cromer [3] allows all of $R, K$ and $E$ to vary periodically and uses a perturbation method to obtain a range of results about $\mathrm{P}(\mathrm{T})$.

Our approach here is different to those above, although there are aspects in common. While we do not consider $\mathrm{R}, \mathrm{K}$ and $\mathrm{E}$ to be randomly varying (so that the problem (1) is deterministic) we do take the view that in realistic populations, these parameters will show variation on a much longer time scale to that intrinsic to the population itself. This approach allows us to recast (1) as a problem involving two time scales and to use a multiscaling approach to obtain approximate expressions for $\mathrm{P}(\mathrm{T})$ for arbitrary slowly varying $R, K$ and $E$.

To reformulate (1), we first write it in a dimensionless form. We assume that $R, K$ and $E$ vary on some intrinsic time scale, $T^{*}$; and that they may be expressed in the form

$$
\mathrm{R}(\mathrm{T})=\mathrm{R}_{0} \mathrm{r}\left(\mathrm{T} / \mathrm{T}^{*}\right), \quad \mathrm{K}(\mathrm{T})=\mathrm{K}_{0} \mathrm{k}\left(\mathrm{T} / \mathrm{T}^{*}\right), \quad \mathrm{E}(\mathrm{T})=\mathrm{E}_{0} \mathrm{e}\left(\mathrm{T} / \mathrm{T}^{*}\right)
$$

respectively, where $R_{0}, K_{0}$ and $E_{0}$ are characteristic values of the respective quantities. Such values might typically be a finite upper bound for the magnitude of the quantity on $\mathrm{T}>0$ (when such exists); or the value of that quantity at a specific $\mathrm{T}$ value; for example $\mathrm{T}=0$. Thus, $\mathrm{r}, \mathrm{k}$ and $\mathrm{e}$ are dimensionless functions of their dimensionless arguments. Note that $R(T)$ has the dimension of reciprocal time, so that $R_{0}^{-1}$ has dimension of of time, 
and thus is a natural time scale for the variation of the population. Similarly, $\mathrm{K}_{0}$ has the dimension of population (or population density). This motivates us to define dimensionless variables

$$
\mathrm{t}=\mathrm{R}_{0} \mathrm{~T}, \text { and } \mathrm{p}=\mathrm{P} / \mathrm{K}_{0},
$$

so that the problem (1) converts to the dimensionless form

$$
\frac{d p(t, \varepsilon)}{d t}=r(\varepsilon t) p(t, \varepsilon)\left(1-\frac{p(t, \varepsilon)}{k(\varepsilon t)}\right)-\mu e(\varepsilon t) p(t, \varepsilon), \quad p(t=0)=p_{0}
$$

where

$$
\varepsilon=\frac{R_{0}^{-1}}{T^{*}}, \quad \mu=\frac{E_{0}}{R_{0}} \quad \text { and } \quad p_{0}=\frac{P_{0}}{K_{0}}
$$

are dimensionless ratios of characteristic values. In particular, $\varepsilon$ measures the ratio of the time scale for population growth to the time scale intrinsic to $R(T), K(T)$ and $E(T)$. Note that (4) displays $p$ as $p(t, \varepsilon)$, showing variation with respect to $t$, with $\varepsilon$ as a parameter.

In what follows, we regard $\varepsilon$ to be small; that is, the time scale for variation of $R, K$ and $E$ is long relative to $R_{0}^{-1}$, the 'natural' time scale for $P(T)$. In this case, $r(\varepsilon t), k(\varepsilon t)$ and $e(\varepsilon t)$ are slowly varying functions of $t$.

While the formal solution of (4) is

$$
p(t, \varepsilon)=\frac{p_{0} \exp \left\{\int_{0}^{\varepsilon t}(r(s)-\mu e(s)) d s\right\}}{p_{0} \int_{0}^{\varepsilon t}\left[\frac{r(w)}{k(w)} \exp \left\{\int_{0}^{w}(r(s)-\mu e(s)) d s\right\}\right] d w+1},
$$

the integrals in (6) cannot usually be evaluated exactly and we opt for an approximation to the solution of the problem using a multiscaling approach, as follows. 


\section{The multiscale harvesting equation}

Assuming $\varepsilon$ to be small, we view the harvesting problem (4) as one involving two time scales, a 'slow' time scale, $\varepsilon$ t, and a 'normal' time scale, $t$. The time scale $\varepsilon t$ is regarded as slow time because it takes an $\mathrm{O}\left(\varepsilon^{-1}\right)$ change in $\mathrm{t}$ to produce an $\mathrm{O}(1)$ change in $\varepsilon$ t.

Based on earlier work on similar problems [6], we introduce the generalised time scales

$$
t_{0}=\frac{1}{\varepsilon} h\left(t_{1}\right) \text { and } t_{1}=\varepsilon t,
$$

where $h\left(t_{1}\right)$ is an arbitrary function of $t_{1}$, to be found. On physical grounds, we require that $h\left(t_{1}\right)$ be positive on $t_{1} \geqslant 0, h^{\prime}\left(t_{1}\right)>0$ on $t_{1} \geqslant 0$, which ensures the mapping from $t_{1}$ to $t_{0}$ is one-one, and $h(0)=0$ which makes $t_{0}=0$ at $t_{1}=t=0$.

Following the multiscaling procedure, we then view $p(t, \varepsilon)$ as a function of these two time scales. Defining

$$
p(t, \varepsilon) \equiv \tilde{p}\left(t_{0}, t_{1}, \varepsilon\right),
$$

and on substituting this into (1), we obtain the multiscaled harvesting equation which corresponds to the differential equation in (4),

$$
h^{\prime}\left(t_{1}\right) D_{0} \tilde{p}+\varepsilon D_{1} \tilde{p}=r\left(t_{1}\right) \tilde{p}\left(1-\frac{\tilde{p}}{k\left(t_{1}\right)}\right)-\mu e\left(t_{1}\right) \tilde{p}
$$

where $D_{0}$ and $D_{1}$ denote partial differential operators taken with respect to $t_{0}$ and $t_{1}$ respectively. Note that this process converts an ordinary differential equation, to a partial one and now (9) displays $\varepsilon$ explicitly, rather than implicitly as in (4), allowing the perturbation approach based on $\varepsilon \rightarrow 0$ to be used. 


\section{$3 \quad$ Perturbation analysis}

We now express $\tilde{p}$ as a Poincaré expansion in $\varepsilon$ :

$$
\tilde{p}\left(t_{0}, t_{1}, \varepsilon\right)=\tilde{p}_{0}\left(t_{0}, t_{1}\right)+\varepsilon \tilde{p}_{1}\left(t_{0}, t_{1}\right)+\varepsilon^{2} \tilde{p}_{2}\left(t_{0}, t_{1}\right)+\cdots .
$$

Substituting the expansion (10) into equation (9) and equating like powers of $\varepsilon$ gives us equations for $\tilde{p}_{0}$ and $\tilde{p}_{1}$ :

$$
h^{\prime}\left(t_{1}\right) D_{0} \tilde{p}_{0}=r\left(t_{1}\right) \tilde{p}_{0}-\frac{r\left(t_{1}\right) \tilde{p}_{0}^{2}}{k\left(t_{1}\right)}-\mu e\left(t_{1}\right) \tilde{p}_{0}
$$

and

$$
h^{\prime}\left(t_{1}\right) D_{0} \tilde{p}_{1}+D_{1} \tilde{p}_{0}=r\left(t_{1}\right) \tilde{p}_{1}-\frac{2 r\left(t_{1}\right) \tilde{p}_{0} \tilde{p}_{1}}{k\left(t_{1}\right)}-\mu e\left(t_{1}\right) \tilde{p}_{1}
$$

respectively.

Solving (11) gives

$$
\tilde{p}_{0}=\frac{k\left(t_{1}\right) \theta\left(t_{1}\right) h^{\prime}\left(t_{1}\right)}{r\left(t_{1}\right)+A\left(t_{1}\right) k\left(t_{1}\right) \theta\left(t_{1}\right) h^{\prime}\left(t_{1}\right) e^{-\theta\left(t_{1}\right) t_{0}}},
$$

where $A\left(t_{1}\right)$ is an arbitrary function of $t_{1}$ and

$$
\theta\left(t_{1}\right)=\frac{r\left(t_{1}\right)-\mu e\left(t_{1}\right)}{h^{\prime}\left(t_{1}\right)} .
$$

On rearrangement, (12) becomes a linear equation for $\tilde{p}_{1}$ in terms of $t_{0}$ :

$$
D_{0} \tilde{p}_{1}+\frac{\tilde{p}_{1}}{h^{\prime}\left(t_{1}\right)}\left[-r\left(t_{1}\right)+\frac{2 r\left(t_{1}\right) \tilde{p}_{0}}{k\left(t_{1}\right)}+\mu e\left(t_{1}\right)\right]=-\frac{1}{h^{\prime}\left(t_{1}\right)} D_{1} \tilde{p}_{0} .
$$

We note that $\tilde{p}_{0}$ contains one arbitrary function of $t_{1}$, so we only seek a particular solution of (15) to represent $\tilde{p}_{1}$. Solving (15) gives

$$
\tilde{p}_{1}=\Delta\left[-k^{\prime}\left(t_{1}\right) r\left(t_{1}\right)-\frac{k\left(t_{1}\right) \theta^{\prime}\left(t_{1}\right) r\left(t_{1}\right)}{\theta\left(t_{1}\right)}-\frac{k\left(t_{1}\right) h^{\prime \prime}\left(t_{1}\right) r\left(t_{1}\right)}{h^{\prime}\left(t_{1}\right)}+k\left(t_{1}\right) r^{\prime}\left(t_{1}\right)\right.
$$




$$
\left.+\left(A^{\prime}\left(t_{1}\right) t_{0}-\frac{1}{2} \theta^{\prime}\left(t_{1}\right) A\left(t_{1}\right) t_{0}^{2}\right) h^{\prime}\left(t_{1}\right) k\left(t_{1}\right)^{2} \theta\left(t_{1}\right)^{2} e^{-\theta\left(t_{1}\right) t_{0}}\right]
$$

where $\Delta=\left[r\left(t_{1}\right)+A\left(t_{1}\right) k\left(t_{1}\right) \theta\left(t_{1}\right) h^{\prime}\left(t_{1}\right) e^{-\theta\left(t_{1}\right) t_{0}}\right]^{-1}$. If we consider the $t_{0}$ dependence of $\tilde{p}_{0}$ and $\tilde{p}_{1}$ (so that $t_{1}$ and hence $\theta\left(t_{1}\right)$ are fixed), we see that as $t_{0} \rightarrow \infty$, their behaviour is governed by the exponential $e^{-\theta\left(t_{1}\right) t_{0}}$. The exception to this occurs in the $t_{0} e^{-\theta\left(t_{1}\right) t_{0}}$ and $t_{0}^{2} e^{-\theta\left(t_{1}\right) t_{0}}$ terms in (16). We eliminate these by choosing $A\left(t_{1}\right)$ and $\theta\left(t_{1}\right)$ to be constants. We now consider the consequences of these choices.

\subsection{Subcritical harvesting}

For subcritical harvesting, $r\left(t_{1}\right)-\mu e\left(t_{1}\right)>0$, while since $h^{\prime}\left(t_{1}\right)>0, \theta\left(t_{1}\right)$ is a positive function, from (14), and a constant, from the arguments above. We thus choose $\theta\left(t_{1}\right)=1$, giving

$$
h^{\prime}\left(t_{1}\right)=r\left(t_{1}\right)-\mu e\left(t_{1}\right), \quad h\left(t_{1}\right)=\int_{0}^{t_{1}}(r(s)-\mu e(s)) d s
$$

and leading to the timescales

$$
t_{0}=\frac{1}{\varepsilon} \int_{0}^{t_{1}}(r(s)-\mu e(s)) d s \text { and } t_{1}=\varepsilon t .
$$

Applying the above choices to (13) and (16) we obtain an explicit form of the expansion (10),

$$
\begin{aligned}
& \tilde{p}\left(t_{0}, t_{1}, \varepsilon\right)=\frac{k\left(t_{1}\right) h^{\prime}\left(t_{1}\right)}{r\left(t_{1}\right)+c k\left(t_{1}\right) h^{\prime}\left(t_{1}\right) e^{-t_{0}}} \\
& -\varepsilon \frac{k^{\prime}\left(t_{1}\right) r\left(t_{1}\right) h^{\prime}\left(t_{1}\right)+k\left(t_{1}\right) h^{\prime \prime}\left(t_{1}\right) r\left(t_{1}\right)-k\left(t_{1}\right) r^{\prime}\left(t_{1}\right) h^{\prime}\left(t_{1}\right)}{h^{\prime}\left(t_{1}\right)\left(r\left(t_{1}\right)+c k\left(t_{1}\right) h^{\prime}\left(t_{1}\right) e^{-t_{0}}\right)^{2}}
\end{aligned}
$$




$$
+\cdots
$$

where $h^{\prime}\left(t_{1}\right)$ is given by (17), $t_{0}$ is defined by (18) and $c$ is a constant.

Since our expansion consists of both leading order terms and $\mathrm{O}(\varepsilon)$ terms, we assume that our constant $\mathrm{c}=\mathrm{c}_{0}+\varepsilon \mathrm{c}_{1}+\cdots$.

On applying the initial condition $\tilde{p}(0,0, \varepsilon)=p_{0}$ to our expansion (19), we obtain

$$
\begin{aligned}
p_{0}= & \frac{k_{0} h_{0}^{\prime}}{r_{0}+\left(c_{0}+\varepsilon c_{1}+\cdots\right) k_{0} h_{0}^{\prime}} \\
& -\varepsilon \frac{k_{0}^{\prime} r_{0} h_{0}^{\prime}+k_{0} h_{0}^{\prime \prime} r_{0}-k_{0} r_{0}^{\prime} h_{0}^{\prime}}{h_{0}^{\prime}\left(r_{0}+\left(c_{0}+\varepsilon c_{1}+\cdots\right) k_{0} h_{0}^{\prime}\right)^{2}}+\cdots,
\end{aligned}
$$

where zero subscripts denote values at $t=0$. By expanding (20) in powers of $\varepsilon$, then solving for $\mathrm{c}_{0}$ and $\mathrm{c}_{1}$, we find

$$
\begin{aligned}
c_{0} & =\frac{k_{0} h_{0}^{\prime}-p_{0} r_{0}}{p_{0} k_{0} h_{0}^{\prime}} \\
\text { and } \quad c_{1} & =\frac{h_{0}^{\prime} k_{0} r_{0}^{\prime}-k_{0} h_{0}^{\prime \prime} r_{0}-k_{0}^{\prime} r_{0} h_{0}^{\prime}}{h_{0}^{\prime 3} k_{0}^{2}} .
\end{aligned}
$$

Now our two term expansion for the solution of the non-dimensional harvesting problem becomes

$$
\begin{aligned}
p(t, \varepsilon)= & \frac{k(\varepsilon t) h^{\prime}(\varepsilon t) p_{0} k_{0} h_{0}^{\prime}}{\Psi} \\
& -\varepsilon \frac{p_{0}^{2}}{h^{\prime}(\varepsilon t) h_{0}^{\prime} \Psi^{2}}\left\{h^{\prime}(\varepsilon t)^{3} k(\varepsilon t)^{2} A e^{-t_{0}}+k_{0}^{2} h_{0}^{\prime 3} B(\varepsilon t)\right\}+\cdots(23)
\end{aligned}
$$

where

$$
\begin{aligned}
\Psi & =r(\varepsilon t) p_{0} k_{0} h_{0}^{\prime}+k(\varepsilon t) h^{\prime}(\varepsilon t)\left(k_{0} h_{0}^{\prime}-r_{0} p_{0}\right) e^{-t_{0}}, \\
A & =k_{0} r_{0}^{\prime} h_{0}^{\prime}-k_{0}^{\prime} r_{0} h_{0}^{\prime}-k_{0} h_{0}^{\prime \prime} r_{0},
\end{aligned}
$$




$$
B(\varepsilon t)=k^{\prime}(\varepsilon t) r(\varepsilon t) h^{\prime}(\varepsilon t)-k(\varepsilon t) r^{\prime}(\varepsilon t) h^{\prime}(\varepsilon t)+k(\varepsilon t) h^{\prime \prime}(\varepsilon t) r(\varepsilon t),
$$

while $h^{\prime}(\varepsilon t)$ is given by (17) and $t_{0}$ is defined by (18). Equation (23) now provides a two term approximation to the solution of the harvesting model when the growth rate, carrying capacity and effort vary slowly with time and the harvesting is subcritical.

From (23) we can see that as time $t \rightarrow \infty$ (and hence $t_{0} \rightarrow \infty$ ),

$$
\begin{aligned}
p(t, \varepsilon) \rightarrow & \frac{k(\varepsilon t)(r(\varepsilon t)-\mu e(\varepsilon t))}{r(\varepsilon t)} \\
& -\varepsilon\left(\frac{k^{\prime}(\varepsilon t)}{r(\varepsilon t)}-\frac{k(\varepsilon t) r^{\prime}(\varepsilon t)}{r(\varepsilon t)^{2}}+\frac{k(\varepsilon t)\left(r^{\prime}(\varepsilon t)-\mu e^{\prime}(\varepsilon t)\right)}{r(\varepsilon t)(r(\varepsilon t)-\mu e(\varepsilon t))}\right) \\
& +\cdots .
\end{aligned}
$$

Hence, over time, the population tends to the limiting value of $k(\varepsilon t)(r(\varepsilon t)-$ $\mu e(\varepsilon t)) / r(\varepsilon t)$ minus an $\mathrm{O}(\varepsilon)$ correction. This gives a two term approximation to the slowly varying limiting population.

\subsection{Supercritical harvesting}

Here, $r\left(t_{1}\right)-\mu e\left(t_{1}\right)<0$, on $t_{1} \geqslant 0$, that is the harvesting term exceeds the critical value $r(t)$ for all time. However, while we still choose $A\left(t_{1}\right)$ to be a constant, $c$, we now choose $\theta\left(t_{1}\right)=-1$, to preserve the condition that $h^{\prime}\left(t_{1}\right)>0$. This then gives

$$
h^{\prime}\left(t_{1}\right)=\mu e\left(t_{1}\right)-r\left(t_{1}\right), \quad h\left(t_{1}\right)=\int_{0}^{t_{1}}(\mu e(s)-r(s)) d s
$$

so that

$$
t_{0}=-\frac{1}{\varepsilon} \int_{0}^{t_{1}}(r(s)-\mu e(s)) d s \text { and } t_{1}=\varepsilon t
$$


Since again, in this case, $t_{0}=0$ at $t=0$, the calculation of the constant $c$ proceeds as before, with $\boldsymbol{c}_{0}$ and $\boldsymbol{c}_{1}$ being given again by (21) and (22). Thus our expansion for $p(t, \varepsilon)$ on $t \geqslant 0$ becomes

$$
\begin{aligned}
p(t, \varepsilon)= & \frac{k(\varepsilon t) h^{\prime}(\varepsilon t) p_{0} k_{0} h_{0}^{\prime}}{\Psi^{*}} \\
& -\varepsilon \frac{p_{0}^{2}}{h^{\prime}(\varepsilon t) h_{0}^{\prime} \Psi^{* 2}}\left\{h^{\prime}(\varepsilon t)^{3} k(\varepsilon t)^{2} A e^{t_{0}}+k_{0}^{2} h_{0}^{\prime 3} B(\varepsilon t)\right\}+\cdots
\end{aligned}
$$

where

$$
\Psi^{*}=r(\varepsilon t) p_{0} k_{0} h_{0}^{\prime}+k(\varepsilon t) h^{\prime}(\varepsilon t)\left(k_{0} h_{0}^{\prime}-r_{0} p_{0}\right) e^{t_{0}},
$$

and $t_{0}$ is defined by (26). $A$ and $B(\varepsilon t)$ are as defined in (23), with $h^{\prime}\left(t_{1}\right)$ given by (25). Note that now, $p(t, \varepsilon) \rightarrow 0$ as $t \rightarrow \infty$; that is, the population is driven to extinction, but as expected, this extinction takes infinite time.

\section{Comparison with numerical solutions}

Here we compare the results (23) and (27) with the corresponding numerical solutions of (4). We begin by considering the case where both $r$ and $k$ are constant and only the effort $e$ varies slowly and periodically, that is,

$$
k(\varepsilon t) \equiv 1, \quad r(\varepsilon t) \equiv 1, \quad e(\varepsilon t)=0.5+0.24 \cos \varepsilon t, \quad \mu=0.2 .
$$

Here, $r-\mu e=0.9-0.048 \cos \varepsilon t$, and the harvesting is subcritical on $t \geqslant 0$. Figure 1 compares the result obtained by using the expansion (23) with that obtained by numerically solving (4), when $\varepsilon=0.1$ and $p_{0}=0.1$. The approximation and numerical results virtually, with both showing a rapid transition from the starting population to a periodic equilibrium state, given by $(24)$. 


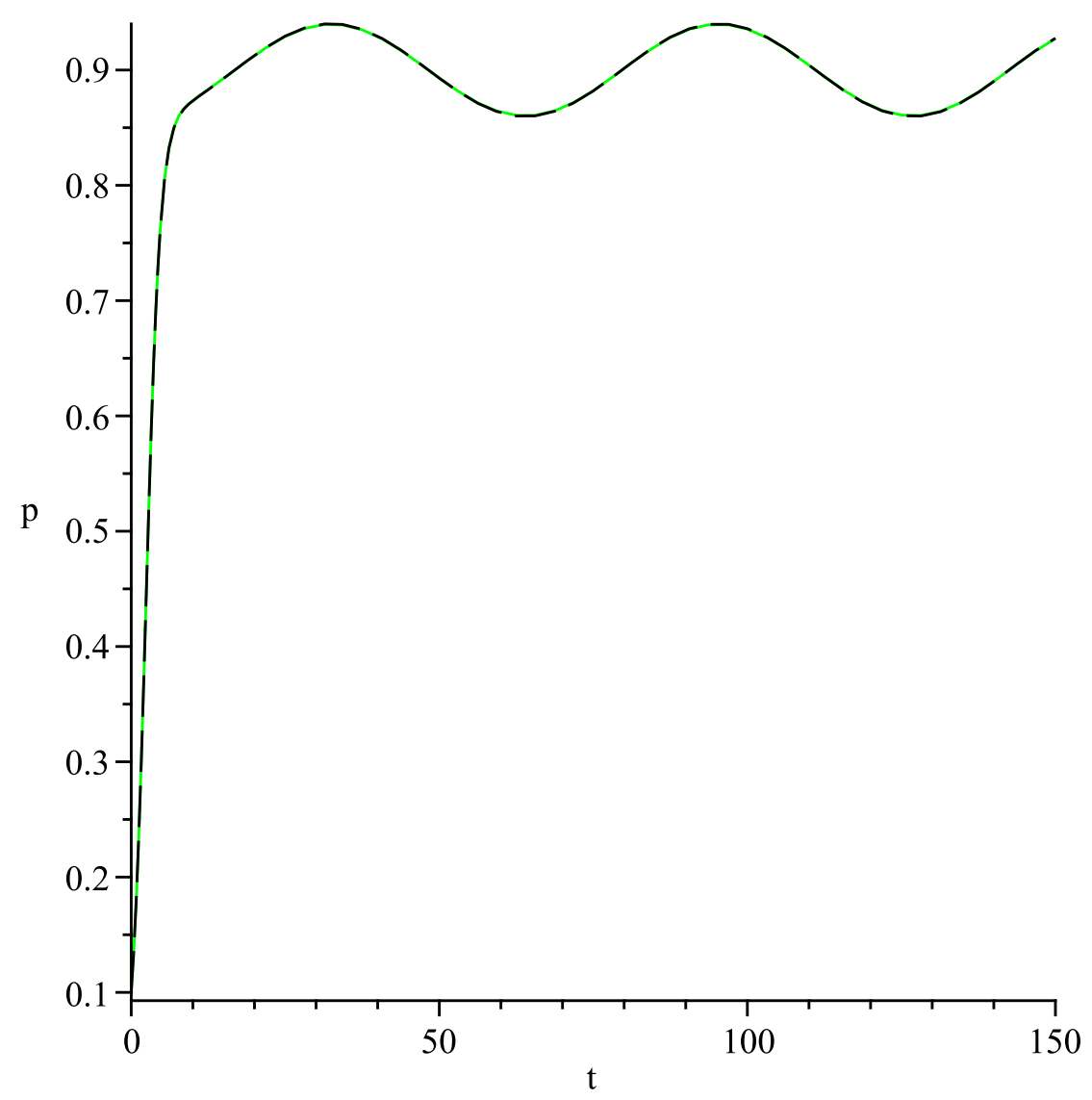

Figure 1: Population, $\mathrm{p}$, as a function of time, $\mathrm{t}$, for harvesting, where the multi-scale approximation (23) is shown as (black-dashed)and numerical solution as (green-solid); for the choice of system equations (28), with $p_{0}=$ 0.1 and $\varepsilon=0.1$. Note that the black dashed curve sits almost exactly on top of the green continuous curve. 
Next we consider slowly varying periodic $k$ and $e$ and a constant $r$, namely

$$
k(\varepsilon t)=0.9+0.2 \sin \varepsilon t, \quad r(\varepsilon t) \equiv 1, \quad e(\varepsilon t)=1+0.4 \sin \varepsilon t, \quad \mu=0.05 .
$$

Here, $r-\mu e=0.95-0.02 \sin \varepsilon t$, and again, the harvesting is subcritical. Again, Figure 2 compares the approximation with the results of numerical calculations; both virtually coincide, while again, a periodic equilibrium state is reached.

Finally, we consider constants $k$ and $r$, with $e$ being periodic

$$
k(\varepsilon t) \equiv 1, \quad r(\varepsilon t) \equiv 1, \quad e(\varepsilon t)=1+0.1 \sin \varepsilon t, \quad \mu=2,
$$

so that $r-\mu e=-1-0.2 \sin \varepsilon t$, and now, the harvesting is supercritical. Figure 3 compares the result of using (30) in (27) with numerical solutions of (4), when $\varepsilon=0.1$ and $p_{0}=0.5$. Again, approximation and numerical results effectively coincide. This time, as expected, the population tends to zero.

\section{Discussion}

The two-term expansion (23) and (27) provide explicit, easily evaluated approximate representations of the population $p(t, \varepsilon)$ of $(4)$ in the subcritical and supercritical harvesting cases, respectively, when $\varepsilon$ is small and positive. They apply to any given set of functions $r(\varepsilon t), k(\varepsilon t)$ and $e(\varepsilon t)$, with the only proviso being that these be differentiable. Thus there is no particular restriction to periodic functions that exist in other work [7,3]. When $r, k$ and $e$ are periodic, the analysis of Section 3.1 shows that the limiting state, (24), is periodic, as predicted by Rosenblat [9].

Approximations (23) and (27) are formal results only. To validate them as approximations to the solution of (4) is a calculation well beyond the scope of this paper, although it has been done elsewhere [4]. Nevertheless, the 


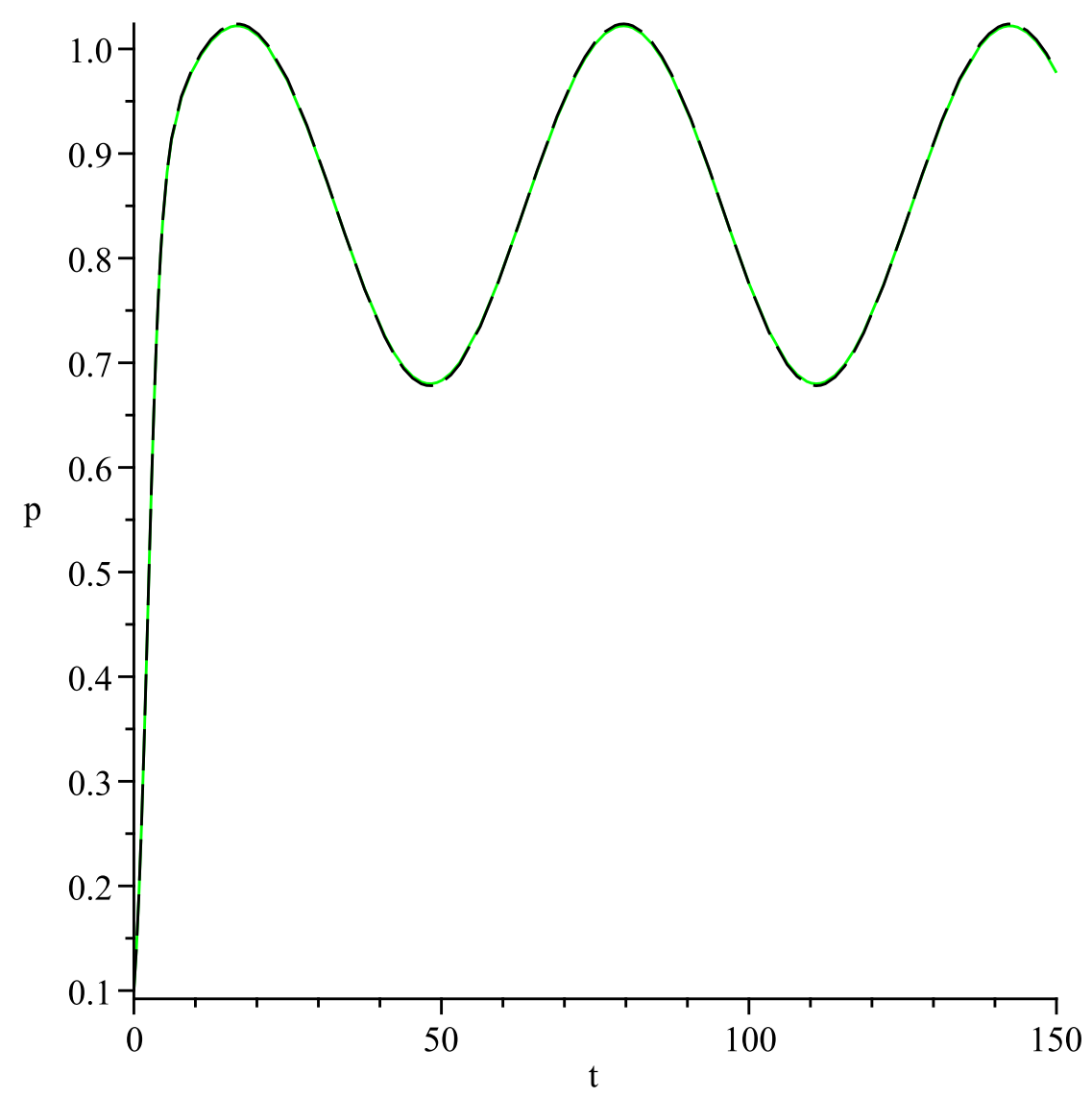

Figure 2: Population, $\mathrm{p}$, as a function of time, $\mathrm{t}$, for harvesting, where the multi-scale approximation (23) is shown as (black-dashed)and numerical solution as (green-solid); for the choice of system equations (29), with $p_{0}=$ 0.1 and $\varepsilon=0.1$. Note that the black dashed curve sits almost exactly on top of the green continuous curve. 


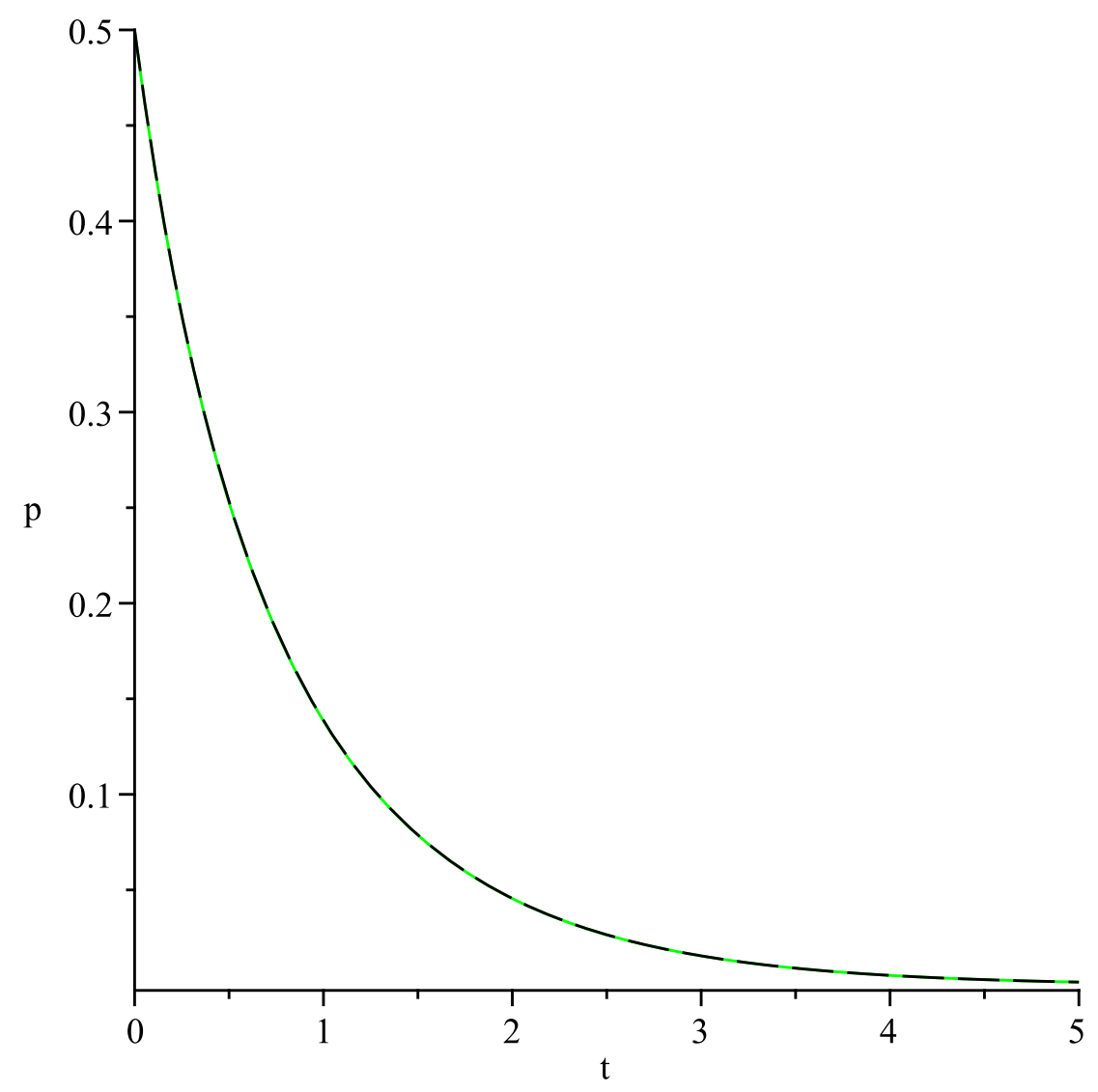

Figure 3: Population, $p$, as a function of time, $t$, for harvesting, where the multi-scale approximation (23) is shown as (black-dashed)and numerical solution as (green-solid); for the choice of functions (30), with $\mathrm{p}_{0}=0.5$ and $\varepsilon=0.1$. Note that the black dashed curve sits almost exactly on top of the green continuous curve. 
excellent agreement with numerical solutions, as depicted in Figures 1, 2 and 3 is encouraging.

Note that $h^{\prime}(\varepsilon t)$ occurs in the denominator of the second term of each of (23) and (27). Thus, at points where $h^{\prime}(\varepsilon t)=r(\varepsilon t)-\mu e(\varepsilon t)$ is $O(\varepsilon),(23)$ and $(27)$ experience disordering, and they fail to represent the population $p(t, \varepsilon)$. This problem of disordering was also noted for the Gompertz model [5]. This phenomenon is most important in neighbourhoods of points where $h^{\prime}(\varepsilon t)=0$, which are the transition points of (4). This will be discussed in a subsequent paper, where the present results will construct an approximation to the evolving population for a transition from a region of subcritical harvesting to one of supercritical harvesting.

\section{References}

[1] J. R. Beddington and R. M. May. Harvesting natural populations in a randomly fluctuating environment. Science, 197:463-465, 1977. C34

[2] C. W. Clark. Mathematical Bioeconomics: The Optimal Management of Renewable Resources. 2nd Edn. Wiley-Interscience, 2005. C33

[3] T. L. Cromer. Harvesting in a seasonal environment. Math. Comput. Model., 10(6):445-450, 1988. C34, C43

[4] T. Grozdanovski. Multi-Scaling Methods Applied to Population Models. Ph.D. Thesis, RMIT University, 2009. C43

[5] T. Grozdanovski and J. J. Shepherd. Slow variation in the Gompertz model. ANZIAM J., 47:C451-C554, 2007. http://anziamj.austms . org.au/ojs/index.php/ANZIAMJ/article/view/1061 C46

[6] T. Grozdanovski, J. J. Shepherd and A. Stacey. Multiscaling analysis of a logistic model with slowly varying coefficients. Appl. Math. Lett., 22:1091-1095, Elsevier, 2009. doi:10.1016/j.aml.2008.10.002. C36 
[7] T. Legovic and G. Peric. Harvesting population in a periodic environment. Ecol. Model., 24:221-229, 1984. C34, C43

[8] J. D. Murray. Mathematical Biology I: An Introduction. 3rd Edn. Springer-Verlag, Berlin, 2007. C33

[9] S. Rosenblat. Population models in a periodically fluctuating environment. J. Math. Biol., 9:23-36, 1980. C34, C43

\section{Author addresses}

1. T. Grozdanovski, School of Mathematical and Geospatial Sciences, RMIT University, Melbourne, Victoria 3001, Australia.

2. J. J. Shepherd, School of Mathematical and Geospatial Sciences, RMIT University, Melbourne, Victoria 3001, Australia. mailto: jshep@rmit.edu.au

3. A. Stacey, School of Mathematical and Geospatial Sciences, RMIT University, Melbourne, Victoria 3001, Australia. 